\title{
On the Fourth Power Mean of the Two-Term Exponential Sums
}

\author{
Han Zhang and Wenpeng Zhang \\ Department of Mathematics, Northwest University, Xian, Shaanxi, China \\ Correspondence should be addressed to Wenpeng Zhang; wpzhang@nwu.edu.cn \\ Received 17 October 2013; Accepted 18 December 2013; Published 12 January 2014 \\ Academic Editors: C.-S. Liu and C. Zhai
}

Copyright (C) $2014 \mathrm{H}$. Zhang and W. Zhang. This is an open access article distributed under the Creative Commons Attribution License, which permits unrestricted use, distribution, and reproduction in any medium, provided the original work is properly cited.

The main purpose of this paper is to use the analytic methods and the properties of Gauss sums to study the computational problem of one kind fourth power mean of two-term exponential sums and give an interesting identity and asymptotic formula for it.

\section{Introduction}

Let $q \geq 3$ be a positive integer. For any integers $m$ and $n$, the two-term exponential sum $C(m, n, k ; q)$ is defined as follows:

$$
C(m, n, k ; q)=\sum_{a=1}^{q} e\left(\frac{m a^{k}+n a}{q}\right)
$$

where $e(y)=e^{2 \pi i y}$.

Regarding the various properties of $C(m, n, k ; q)$, some authors have studied them, and obtained a series of results. Some related works can be found in references [1-8]. For example, Gauss' classical work proved the remarkable formula (see [1])

$$
\begin{aligned}
C(1,0,2 ; q) & =\frac{1}{2} \sqrt{q}(1+i)\left(1+e\left(-\frac{q}{4}\right)\right) \\
& = \begin{cases}\sqrt{q}, & \text { if } q \equiv 1 \bmod 4, \\
0, & \text { if } q \equiv 2 \bmod 4 \\
i \sqrt{q}, & \text { if } q \equiv 3 \bmod 4 \\
(1+i) \sqrt{q}, & \text { if } q \equiv 0 \bmod 4\end{cases}
\end{aligned}
$$

where $i^{2}=-1$.

Generally, for any odd number $q$ and $(m, q)=1$, the exact value of $|C(m, 0,2 ; q)|$ is $\sqrt{q}$, More relevant to it, Cochrane and Zheng [4] showed the general sum that

$$
|C(m, n, k ; q)| \leq k^{\omega(q)} q^{1 / 2},
$$

where $\omega(q)$ denotes the number of all distinct prime divisors of $q$.
In this paper, we study the fourth power mean of the twoterm exponential sum $C(m, n, k ; q)$ as follows:

$$
\sum_{m=1}^{q}|C(m, n, k ; q)|^{4},
$$

where $n$ is any integer with $(n, q)=1$.

Regarding this problem, it seems that none has studied it yet; at least we have not seen any related result before. The problem is interesting, because it can reflect that the mean value of $C(m, n, k ; q)$ is well behaved. The main purpose of this paper is to use the analytic methods and the properties of Gauss sums to study the special case of (4) with $k=5, q=p$, an odd prime and give an interesting identity and asymptotic formula for it. That is, we will prove the following conclusion.

Theorem 1. Let $p>3$ be a prime. Then for any integer $n$ with $(n, p)=1$, one has the identity

$$
\begin{aligned}
\sum_{m=1}^{p} & \left|\sum_{a=1}^{p-1} e\left(\frac{m a^{5}+n a}{p}\right)\right|^{4} \\
& = \begin{cases}3 p^{3}-p^{2}\left(8+2\left(\frac{-1}{p}\right)+4\left(\frac{-3}{p}\right)\right)-3 p, & \text { if } 5 \dagger p-1, \\
3 p^{3}+O\left(p^{2}\right), & \text { if } 5 \mid p-1,\end{cases}
\end{aligned}
$$

where $(* / p)$ denotes the Legendre symbol. 
For any prime $p$ with $5 \mid p-1$, one cannot give an exact computational formula in our theorem at present. The difficulty is that one needs to know the value of the character sums

$$
\sum_{i=1}^{4}\left|\sum_{a=1}^{p-1} \chi_{1}^{i}\left(a^{5}-1\right)\right|^{2}
$$

where $\chi_{1}$ is any 5-order character $\bmod p$.

For any integer $h \geq 3$, whether there exists an exact computational formula for

$$
\sum_{m=1}^{p}\left|\sum_{a=1}^{p-1} e\left(\frac{m a^{5}+n a}{p}\right)\right|^{2 h}
$$

is an open problem, where $p$ is an odd prime and $(n, p)=1$.

\section{Several Lemmas}

In this section, we will give several lemmas which are necessary in the proof of our theorem. In the proving process of all lemmas, we used many properties of Gauss sums; all these can be found in [1], so they will not be repeated here. First we have the following.

Lemma 2. Letting $p$ be an odd prime with $p>3$, then one has the identity

$$
\sum_{\substack{a=1 \\ p \mid(a-b)(a+b+1)\left(a^{2}+b^{2}+a+b+1\right)}}^{p-2} 1=3 p-10-2\left(\frac{-1}{p}\right)-4\left(\frac{-3}{p}\right),
$$

where $(* / p)$ denotes the Legendre's symbol.

Proof. For any prime $p$, note that if $a$ passes through a complete residue system mod $p$, then $2 a+1$ also passes through a complete residue system $\bmod p$, so note the identity

$$
\sum_{a=1}^{p}\left(\frac{a^{2}+n}{p}\right)= \begin{cases}-1, & \text { if }(n, p)=1 \\ p-1, & \text { if }(n, p)=p\end{cases}
$$

(this formula can be found in Hua's book, Section 7.8, Theorem 8.2 [9]). One has

$$
\begin{aligned}
& \sum_{a=1}^{p-2} \sum_{b=1}^{p-2} 1 \\
& p \mid(a-b)(a+b+1)\left(a^{2}+b^{2}+a+b+1\right) \\
& =\sum_{\substack{a=1 \\
p \mid(a-b)(a+b+1) \\
p \dagger a^{2}+b^{2}+a+b+1}}^{p-2} \sum_{\substack{a-1 \\
p \dagger(a-b)(a+b+1) \\
p \mid a^{2}+b^{2}+a+b+1}}^{p-2} 1+\sum_{\substack{a=1 \\
p|(a-b)(a+b+1) \\
p| a^{2}+b^{2}+a+b+1}}^{p-2} \sum_{b=1}^{p-2} 1 \\
& =2(p-2)-1-\sum_{\substack{a=1 \\
p \mid 2 a^{2}+2 a+1}}^{p-2} 1+\sum_{\substack{a=1 \\
p \mid a^{2}+b^{2}+a+b+1}}^{p-2} \sum_{\substack{p=1 \\
p-2}} 1 \\
& =2 p-5-\sum_{\substack{a=1 \\
p \mid(2 a+1)^{2}+1}}^{p-2} 1-4 \sum_{\substack{a=0 \\
p \mid(2 a+1)^{2}+3}}^{p-1} 1 \\
& +\sum_{\substack{a=0 \\
p \mid(2 a+1)^{2}+(2 b+1)^{2}+2}}^{p-1} \sum_{\substack{p-1\\
}}^{p-0} 1
\end{aligned}
$$

$$
\begin{aligned}
= & 2 p-5-\sum_{\substack{a=0 \\
p \mid a^{2}+1}}^{p-1} 1-4 \sum_{\substack{a=0 \\
p \mid a^{2}+3}}^{p-1} 1+\sum_{\substack{a=0 \\
p \mid a^{2}+b^{2}+2}}^{p-1} \sum_{b=0}^{p-1} 1 \\
= & 2 p-5-\left(1+\left(\frac{-1}{p}\right)\right)-4\left(1+\left(\frac{-3}{p}\right)\right) \\
& +\sum_{b=0}^{p-1}\left(1+\left(\frac{-\left(b^{2}+2\right)}{p}\right)\right) \\
= & 3 p+\sum_{b=0}^{p-1}\left(\frac{-\left(b^{2}+2\right)}{p}\right)-10-4\left(\frac{-3}{p}\right)-\left(\frac{-1}{p}\right) \\
= & 3 p-10-2\left(\frac{-1}{p}\right)-4\left(\frac{-3}{p}\right) .
\end{aligned}
$$

This proves Lemma 2.

Lemma 3. Let $p$ be an odd prime $\chi$ be any nonprincipal character $\bmod p$. Then for any integer $n$ with $(n, p)=1$, one has the identity

$$
\begin{aligned}
& \left.\left|\sum_{m=1}^{p-1} \chi(m)\right| \sum_{a=1}^{p-1} e\left(\frac{m a^{5}+n a}{p}\right)\right|^{2} \mid \\
& p \mid \begin{array}{c}
p\left|\sum_{a=1}^{p-2} \bar{\chi}\left(5 a^{4}+10 a^{3}+10 a^{2}+5 a+1\right)\right|, \\
\text { if } \chi \text { is not a } 5 \text {-order character } \bmod p, \\
\sqrt{p} \mid \begin{array}{c}
p-1 \\
-2
\end{array} \sum_{a=1}^{p-1} \bar{\chi}(a(1-a))+\sum_{a=1}^{p-1} \bar{\chi}\left(a^{2}(a-1)\right) \\
\text { if } \chi \text { is a } 5 \text {-order character } \bmod p .
\end{array}
\end{aligned}
$$


Proof. Note that $\chi$ is a non-principal character $\bmod p$, so if $\chi$ is not a 5 -order character $\bmod p$, (i.e., $\chi^{5} \neq \chi_{0}$, the principal character $\bmod p$ ), then from the properties of Gauss sums we have

$$
\begin{aligned}
& \sum_{m=1}^{p-1} \chi(m)\left|\sum_{a=1}^{p-1} e\left(\frac{m a^{5}+n a}{p}\right)\right|^{2} \\
& =\sum_{a=1}^{p-1} \sum_{b=1}^{p-1} \sum_{m=1}^{p-1} \chi(m) e\left(\frac{m\left(a^{5}-b^{5}\right)+n(a-b)}{p}\right) \\
& =\sum_{a=1}^{p-1} \sum_{b=1}^{p-1} \sum_{m=1}^{p-1} \chi(m) e\left(\frac{m b^{5}\left(a^{5}-1\right)+n b(a-1)}{p}\right) \\
& =\tau(\chi) \sum_{a=1}^{p-1} \bar{\chi}\left(a^{5}-1\right) \sum_{b=1}^{p-1} \bar{\chi}^{5}(b) e\left(\frac{n b(a-1)}{p}\right) \\
& =\tau(\chi) \tau\left(\bar{\chi}^{5}\right) \sum_{a=1}^{p-1} \bar{\chi}\left(a^{5}-1\right) \chi^{5}(n(a-1)) \\
& =\chi^{5}(n) \tau(\chi) \tau\left(\bar{\chi}^{5}\right) \sum_{a=1}^{p-2} \bar{\chi}\left((a+1)^{5}-1\right) \chi\left(a^{5}\right) \\
& =\chi^{5}(n) \tau(\chi) \tau\left(\bar{\chi}^{5}\right) \sum_{a=1}^{p-2} \bar{\chi}\left((\bar{a}+1)^{5}-\bar{a}^{5}\right) \\
& =\chi^{5}(n) \tau(\chi) \tau\left(\bar{\chi}^{5}\right) \sum_{a=1}^{p-2} \bar{\chi}\left(5 a^{4}+10 a^{3}+10 a^{2}+5 a+1\right),
\end{aligned}
$$

where $\tau(\chi)=\sum_{a=1}^{p-1} \chi(a) e(a / p)$ denotes the classical Gauss sums.

If $\chi$ is a 5-order character $\bmod p$, then $\chi^{5}=\chi_{0}$; note that for any integer $a$ with $(a, p)=1$, we have $\chi^{4}(a)=\bar{\chi}(a)$, $\chi^{3}(a)=\bar{\chi}^{2}(a), \chi(-1)=-1$, and

$$
\begin{aligned}
\chi^{4} & (a)+\chi^{3}(a)+\chi^{2}(a)+\chi(a)+1 \\
& = \begin{cases}5, & \text { if } a \text { is a } 5 \text { th residue } \bmod p, \\
0, & \text { otherwise. }\end{cases}
\end{aligned}
$$

From the method of proving (12) we have the identity

$$
\begin{aligned}
& \sum_{m=1}^{p-1} \chi(m)\left|\sum_{a=1}^{p-1} e\left(\frac{m a^{5}+n a}{p}\right)\right|^{2} \\
& \quad=\tau(\chi) \sum_{a=1}^{p-1} \bar{\chi}\left(a^{5}-1\right) \sum_{b=1}^{p-1} \bar{\chi}^{5}(b) e\left(\frac{n b(a-1)}{p}\right) \\
& =\tau(\chi) \sum_{a=1}^{p-1} \bar{\chi}\left(a^{5}-1\right) \sum_{b=1}^{p-1} e\left(\frac{n b(a-1)}{p}\right) \\
& =-\tau(\chi) \sum_{a=1}^{p-1} \bar{\chi}\left(a^{5}-1\right)
\end{aligned}
$$

$$
\begin{gathered}
=-\tau(\chi) \sum_{a=1}^{p-1}\left(\chi^{4}(a)+\chi^{3}(a)+\chi^{2}(a)+\chi(a)+1\right) \bar{\chi}(a-1) \\
=-\tau(\chi)\left(2 \sum_{a=1}^{p-1} \bar{\chi}(a-1)+2 \sum_{a=1}^{p-1} \bar{\chi}(\bar{a}(1-\bar{a}))\right. \\
\left.+\sum_{a=1}^{p-1} \bar{\chi}\left(a^{2}(a-1)\right)\right) \\
=-\tau(\chi)\left(-2+2 \sum_{a=1}^{p-1} \bar{\chi}(a(1-a))+\sum_{a=1}^{p-1} \bar{\chi}\left(a^{2}(a-1)\right)\right) .
\end{gathered}
$$

Now note that $|\tau(\chi)|=\sqrt{p}$ if $\chi \neq \chi_{0}$. From (12) and (14) we may immediately deduce Lemma 3.

Lemma 4. Let $p$ be an odd prime and let $\chi$ be a 5 th character $\bmod p$. Then one has the identity

$$
\begin{gathered}
\sum_{a=1}^{p-1} \chi(a(a-1))=\frac{\tau^{2}(\chi)}{\tau\left(\chi^{2}\right)}, \\
\sum_{a=1}^{p-1} \chi\left(a^{2}(a-1)\right)=\frac{\tau(\chi) \tau\left(\chi^{2}\right)}{\tau\left(\chi^{3}\right)} .
\end{gathered}
$$

Therefore

$$
\left|\sum_{a=1}^{p-1} \chi(a(a-1))\right|=\sqrt{p}, \quad\left|\sum_{a=1}^{p-1} \chi\left(a^{2}(a-1)\right)\right|=\sqrt{p} .
$$

Proof. Noting that $\chi(-1)=1$, from the definition and properties of the classical Gauss sums, we have

$$
\begin{aligned}
\tau^{2}(\chi) & =\sum_{a=1}^{p-1} \sum_{b=1}^{p-1} \chi(a) \chi(b) e\left(\frac{a+b}{p}\right) \\
& =\sum_{a=1}^{p-1} \chi(a) \sum_{b=1}^{p-1} \chi^{2}(b) e\left(\frac{b(a+1)}{p}\right) \\
& =\tau\left(\chi^{2}\right) \sum_{a=1}^{p-1} \chi(a) \bar{\chi}\left((a+1)^{2}\right) \\
& =\tau\left(\chi^{2}\right) \sum_{a=2}^{p} \chi(a-1) \bar{\chi}\left(a^{2}\right) \\
& =\tau\left(\chi^{2}\right) \sum_{a=2}^{p-1} \chi((1-\bar{a}) \bar{a})=\tau\left(\chi^{2}\right) \sum_{a=1}^{p-1} \chi(a(1-a))
\end{aligned}
$$

or

$$
\sum_{a=1}^{p-1} \chi(a(a-1))=\frac{\tau^{2}(\chi)}{\tau\left(\chi^{2}\right)}
$$


Similarly, we also have

$$
\begin{aligned}
\tau(\chi) \tau\left(\chi^{2}\right) & =\sum_{a=1}^{p-1} \sum_{b=1}^{p-1} \chi(a) \chi^{2}(b) e\left(\frac{a+b}{p}\right) \\
& =\sum_{a=1}^{p-1} \chi(a) \sum_{b=1}^{p-1} \chi^{3}(b) e\left(\frac{b(a+1)}{p}\right) \\
& =\tau\left(\chi^{3}\right) \sum_{a=1}^{p-1} \chi(a) \bar{\chi}\left((a+1)^{3}\right) \\
& =\tau\left(\chi^{3}\right) \sum_{a=2}^{p} \chi(a-1) \bar{\chi}\left(a^{3}\right) \\
& =\tau\left(\chi^{3}\right) \sum_{a=2}^{p-1} \chi\left((1-\bar{a}) \bar{a}^{2}\right) \\
& =\tau\left(\chi^{3}\right) \sum_{a=1}^{p-1} \chi\left(a^{2}(1-a)\right)
\end{aligned}
$$

or

$$
\sum_{a=1}^{p-1} \chi\left(a^{2}(a-1)\right)=\frac{\tau(\chi) \tau\left(\chi^{2}\right)}{\tau\left(\chi^{3}\right)} .
$$

This proves Lemma 4.

\section{Proof of the Theorem}

In this section, we shall complete the proof of our theorem. First from the orthogonality of characters $\bmod p$ we have

$$
\begin{gathered}
\left.\sum_{\chi \bmod p}\left|\sum_{m=1}^{p-1} \chi(m)\right| \sum_{a=1}^{p-1} e\left(\frac{m a^{5}+n a}{p}\right)\right|^{2} \\
=(p-1) \sum_{m=1}^{p-1}\left|\sum_{a=1}^{p-1} e\left(\frac{m a^{5}+n a}{p}\right)\right|^{4} .
\end{gathered}
$$

On the other hand, if $5 \dagger p-1$, then any non-principal character $\chi$ is not a 5 -order character mod $p$. Note that

$$
\begin{aligned}
& \sum_{m=1}^{p-1} \chi_{0}(m)\left|\sum_{a=1}^{p-1} e\left(\frac{m a^{5}+n a}{p}\right)\right|^{2} \\
& \quad=\sum_{a=1}^{p-1} \sum_{b=1}^{p-1} \sum_{m=1}^{p-1} e\left(\frac{m b^{5}\left(a^{5}-1\right)+n b(a-1)}{p}\right) \\
& =(p-1)^{2}+p-2=p^{2}-p-1 .
\end{aligned}
$$

From (22) and Lemma 3 we have

$$
\begin{aligned}
& \left.\left.\sum_{\chi \bmod p}\left|\sum_{m=1}^{p-1} \chi(m)\right| \sum_{a=1}^{p-1} e\left(\frac{m a^{5}+n a}{p}\right)\right|^{2}\right|^{2} \\
& =\left.\left.\left|\sum_{m=1}^{p-1} \chi_{0}(m)\right| \sum_{a=1}^{p-1} e\left(\frac{m a^{5}+n a}{p}\right)\right|^{2}\right|^{2} \\
& +\left.\left.\sum_{\substack{\chi \bmod \\
\chi \neq \chi_{0}}}\left|\sum_{m=1}^{p-1} \chi(m)\right| \sum_{a=1}^{p-1} e\left(\frac{m a^{5}+n a}{p}\right)\right|^{2}\right|^{2} \\
& =\left(p^{2}-p-1\right)^{2} \\
& +\sum_{\substack{\chi \bmod _{\chi} \\
\chi \neq \chi_{0}}} p^{2} \cdot\left|\sum_{a=1}^{p-2} \bar{\chi}\left(5 a^{4}+10 a^{3}+10 a^{2}+5 a+1\right)\right|^{2} \\
& =\left(p^{2}-p-1\right)^{2} \\
& +p^{2} \sum_{\chi \bmod p}\left|\sum_{a=1}^{p-2} \bar{\chi}\left(5 a^{4}+10 a^{3}+10 a^{2}+5 a+1\right)\right|^{2} \\
& -p^{2}\left(\sum_{a=1}^{p-2} \chi_{0}\left(5 a^{4}+10 a^{3}+10 a^{2}+5 a+1\right)\right)^{2} \\
& =\left(p^{2}-p-1\right)^{2}+p^{2}(p-1) \\
& \times \sum_{a=1}^{p-2} \sum_{\substack{4 \\
a^{4}+2 a^{3}+2 a^{2}+a \equiv b^{4}+2 b^{3}+2 b^{2}+b \bmod p}}^{p-2} 1-p^{2}(p-2)^{2} \\
& =\left(p^{2}-p-1\right)^{2}+p^{2}(p-1)
\end{aligned}
$$

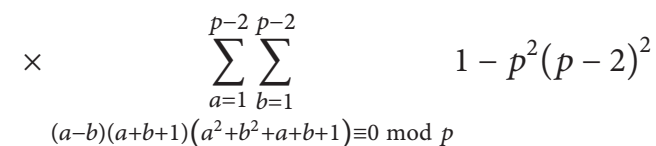

$$
\begin{aligned}
& =\left(p^{2}-p-1\right)^{2}+p^{2}(p-1) \\
& \times\left(3 p-10-2\left(\frac{-1}{p}\right)-4\left(\frac{-3}{p}\right)\right)-p^{2}(p-2)^{2} \\
& =(p-1)\left[3 p^{3}-p^{2}\left(8+2\left(\frac{-1}{p}\right)+4\left(\frac{-3}{p}\right)\right)-3 p-1\right] \text {. }
\end{aligned}
$$

If $5 \dagger p-1$, then combining (21) and (23) we may immediately deduce the identity

$$
\begin{aligned}
& \sum_{m=1}^{p-1}\left|\sum_{a=1}^{p-1} e\left(\frac{m a^{5}+n a}{p}\right)\right|^{4} \\
& \quad=3 p^{3}-p^{2}\left(8+2\left(\frac{-1}{p}\right)+4\left(\frac{-3}{p}\right)\right)-3 p-1
\end{aligned}
$$


or

$$
\begin{aligned}
& \sum_{m=1}^{p}\left|\sum_{a=1}^{p-1} e\left(\frac{m a^{5}+n a}{p}\right)\right|^{4} \\
& \quad=3 p^{3}-p^{2}\left(8+2\left(\frac{-1}{p}\right)+4\left(\frac{-3}{p}\right)\right)-3 p .
\end{aligned}
$$

If $5 \mid p-1$, since $\chi_{1} \neq \chi_{0}$ is a 5 -order character $\bmod p$, $\overline{\chi_{1}}=\chi_{1}^{4}$ and $\bar{\chi}_{1}^{2}=\chi_{1}^{3}$ are also 5-order characters $\bmod p$, then note that

$$
\begin{aligned}
& \sum_{m=1}^{p-1} \chi_{0}(m)\left|\sum_{a=1}^{p-1} e\left(\frac{m a^{5}+n a}{p}\right)\right|^{2} \\
& =(p-1)^{2}-4(p-1)+p-6=p^{2}-5 p-1 \\
& \sum_{a=1}^{p-2} \chi_{1}\left((a+1)^{5}-a^{5}\right) \\
& =\sum_{a=1}^{p-2} \chi_{1}\left((\bar{a}+1)^{5}-1\right)=\sum_{a=1}^{p-1} \chi_{1}\left(a^{5}-1\right) \\
& =\sum_{a=1}^{p-1}\left(\chi_{1}^{2}(a)+\chi_{1}(a)+\bar{\chi}_{1}^{2}(a)+\overline{\chi_{1}}(a)+1\right) \chi_{1}(a-1) \\
& =\sum_{a=1}^{p-1} \chi_{1}\left(a^{2}(1-a)\right)+2 \sum_{a=1}^{p-1} \chi_{1}(a(1-a))-2 .
\end{aligned}
$$

From Lemma 4 and the method of proving (23) we have

$$
\begin{aligned}
& \left.\left.\sum_{\chi \bmod p}\left|\sum_{m=1}^{p-1} \chi(m)\right| \sum_{a=1}^{p-1} e\left(\frac{m a^{5}+n a}{p}\right)\right|^{2}\right|^{2} \\
& =\left(p^{2}-5 p-1\right)^{2} \\
& +\sum_{\substack{\chi \bmod _{\chi} p \\
\chi \neq \chi_{0}, \chi_{1}, \chi_{1}^{2}, \chi_{1}^{3}, \chi_{1}^{4}}} p^{2}\left|\sum_{a=1}^{p-2} \bar{\chi}\left(5 a^{4}+10 a^{3}+10 a^{2}+5 a+1\right)\right|^{2} \\
& +p \sum_{i=1}^{4}\left|\sum_{a=1}^{p-1} \chi_{1}^{i}\left(a^{2}(1-a)\right)+2 \sum_{a=1}^{p-1} \chi_{1}^{i}(a(1-a))-2\right|^{2} \\
& =\left(p^{2}-5 p-1\right)^{2} \\
& +p^{2}(p-1)\left(3 p-10-2\left(\frac{-1}{p}\right)-4\left(\frac{-3}{p}\right)\right) \\
& +\left(p-p^{2}\right) \sum_{i=1}^{4}\left|\sum_{a=1}^{p-1} \chi_{1}^{i}\left(a^{2}(1-a)\right)+2 \sum_{a=1}^{p-1} \chi_{1}^{i}(a(1-a))-2\right|^{2} \\
& -p^{2}(p-6)^{2} \\
& =3 p^{4}+O\left(p^{3}\right) \text {. }
\end{aligned}
$$

So if $5 \mid p-1$, then combining (21) and (27) we can deduce the asymptotic formula

$$
\begin{aligned}
& \left.\sum_{m=1}^{p}|| \sum_{a=1}^{p-1} e\left(\frac{m a^{5}+n a}{p}\right)\right|^{4} \\
& =1+\sum_{m=1}^{p-1}\left|\sum_{a=1}^{p-1} e\left(\frac{m a^{5}+n a}{p}\right)\right|^{4}=3 p^{3}+O\left(p^{2}\right) .
\end{aligned}
$$

Now our theorem follows from (25) and (28).

\section{Conflict of Interests}

The authors declare that there is no conflict of interests regarding the publication of this paper.

\section{Acknowledgments}

The authors express their gratitude to the referee for very helpful and detailed comments. This work is supported by the N. S. F. (11371291), S. R. F. D. P. (20136101110014), and N. S. F. (2013JZ001) of Shaanxi Province of China.

\section{References}

[1] T. M. Apostol, Introduction to Analytic Number Theory, Springer, New York, NY, USA, 1976.

[2] T. M. Apostol, "An extension of the Lehmers' picturesque exponential sums," Mathematics of Computation, vol. 61, no. 203, pp. 25-28, 1993.

[3] B. C. Berndt, "On Gaussian sums and other exponential sums with periodic coefficients," Duke Mathematical Journal, vol. 40, no. 1, pp. 145-156, 1973.

[4] T. Cochrane and Z. Zheng, "Bounds for certain exponential sums," Asian Journal of Mathematics, vol. 4, no. 4, pp. 757-774, 2000 .

[5] T. Cochrane and C. Pinner, "Using Stepanov's method for exponential sums involving rational functions," Journal of Number Theory, vol. 116, no. 2, pp. 270-292, 2006.

[6] K. S. Williams, "Exponential sums over GF(2n)," Pacific Journal of Mathematics, vol. 40, no. 2, pp. 511-519, 1972.

[7] A. Weil, "On some exponential sums," Proceedings of the National Academy of Sciences of the United States of America, vol. 34, no. 5, pp. 204-207, 1948.

[8] T. Wang, "On the fourth power mean of generalized two-term exponential sums," Bulletin of the Korean Mathematical Society, vol. 50, pp. 233-240, 2013.

[9] L. K. Hua, Introducetion to Number Theory, Science Press, Beijing, China, 1957. 


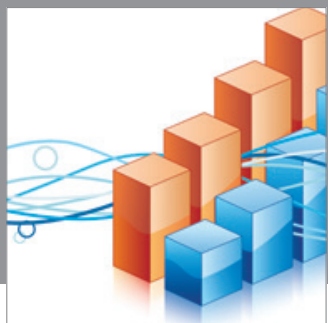

Advances in

Operations Research

mansans

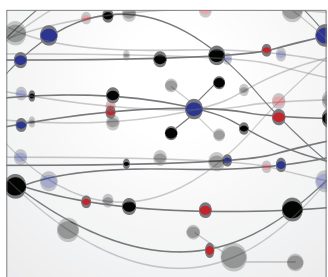

The Scientific World Journal
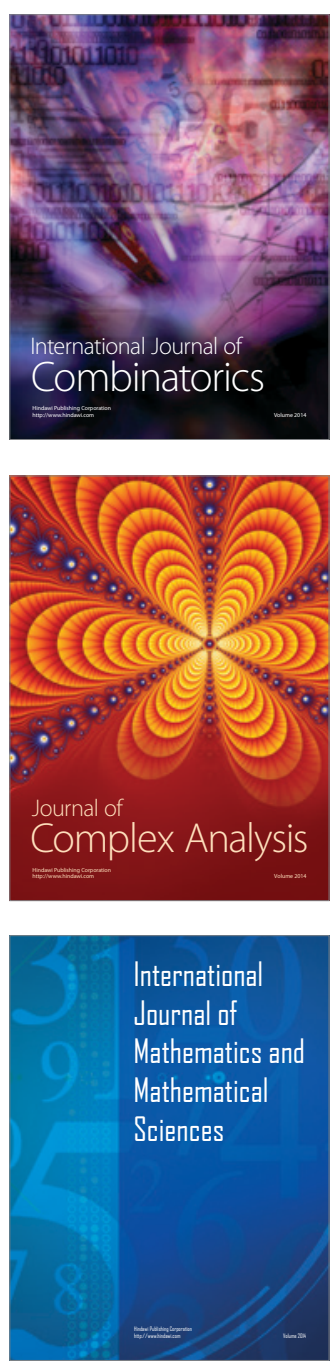
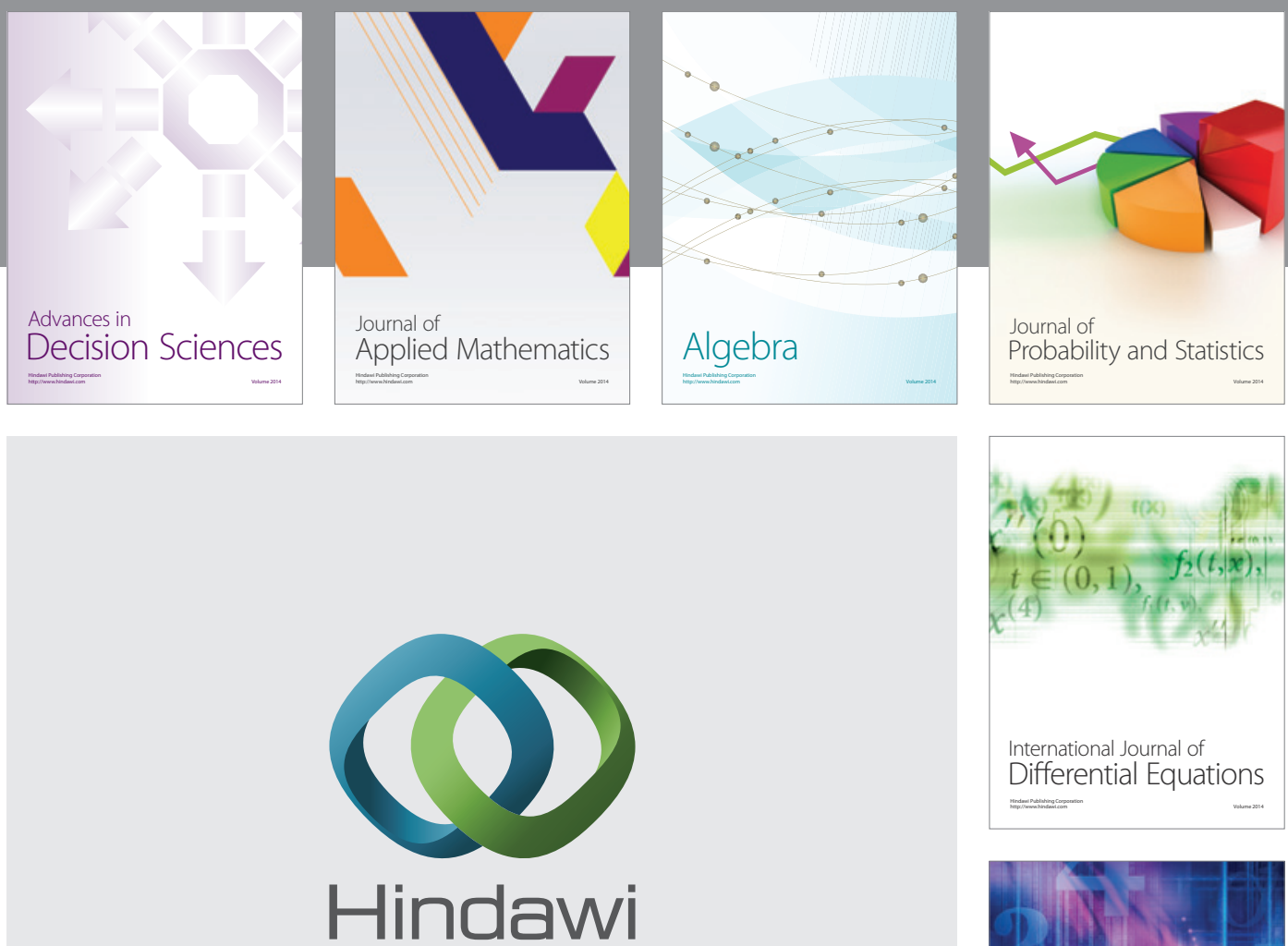

Submit your manuscripts at http://www.hindawi.com
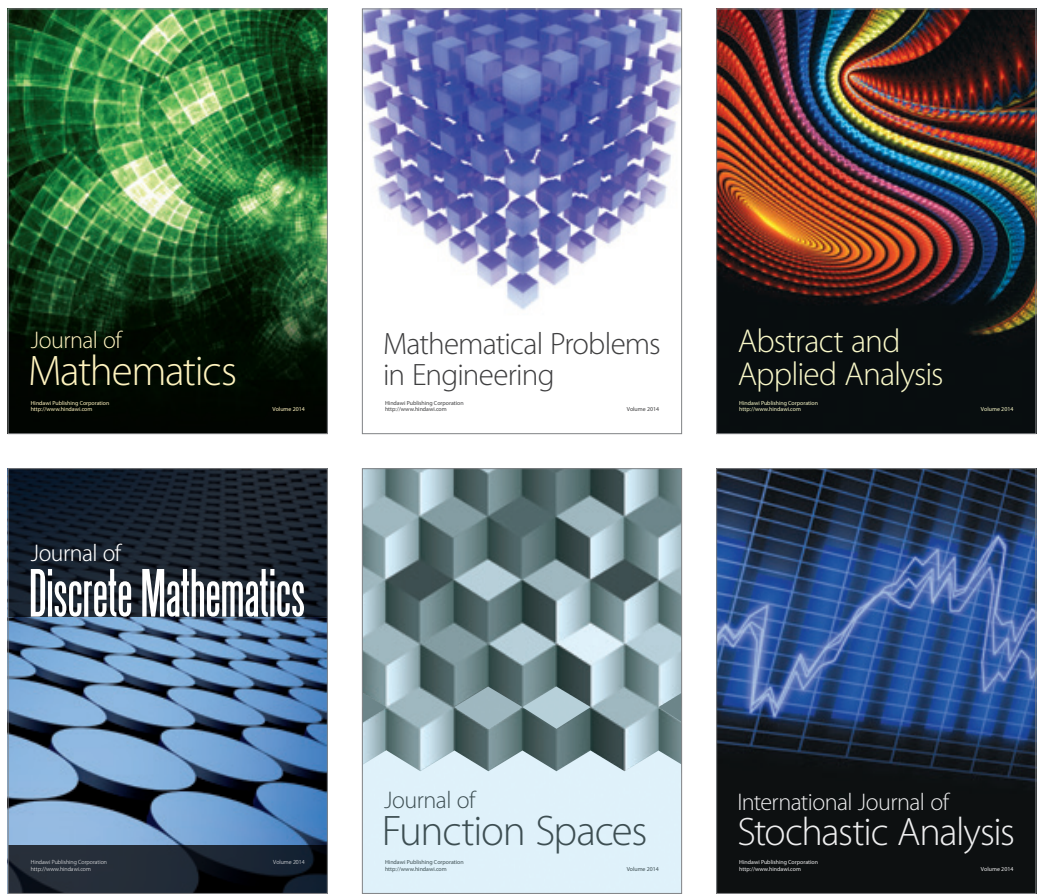

Journal of

Function Spaces

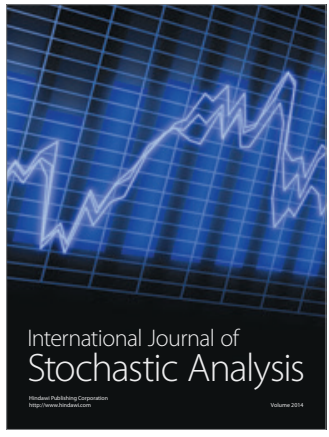

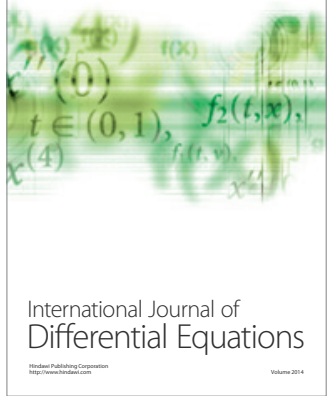
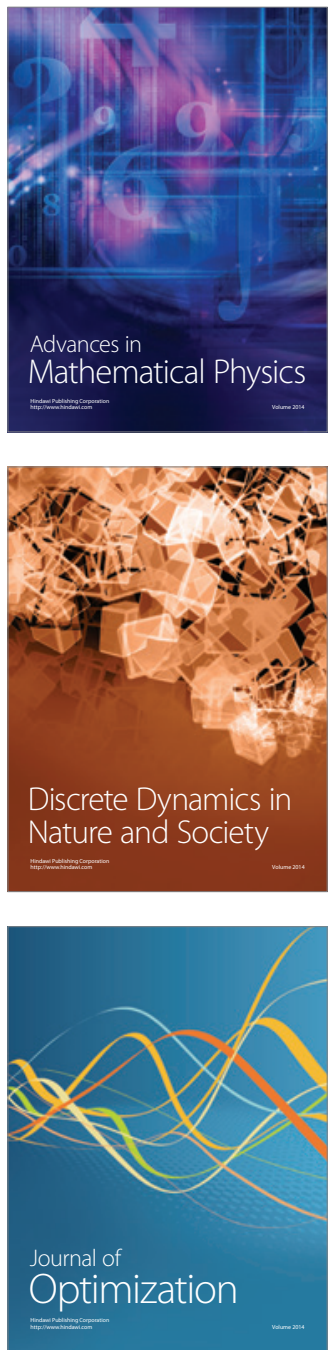